\title{
Custom made ocular prosthesis: A case report
}

Devkota $\mathrm{D}^{1}$, Mathema SRB ${ }^{2}$

${ }^{1}$ Prosthodontist, Cosmetic Dental Clinic, Kathmandu, Nepal.

${ }^{2}$ Professor and HOD, Department of Prosthodontics and Maxillofacial Prosthetics, People's Dental College and Hospital, Kathmandu, Nepal.

\begin{abstract}
Physical defects that compromise appearance or function, which prevent an individual from leading a normal life, usually prompt the individual to seek treatment that will reinstate acceptable normalcy. The loss of an eye impairs the patient's visual function, yet also results in a noticeable deformity. Prosthesis should be provided as soon as possible to raise the spirits and ease the mind of the afflicted. An ocular prosthesis is a maxillofacial prosthesis that artificially replaces an eye missing as a result of trauma, surgery, or congenital absence. A custom ocular prosthesis has advantage of close adaptation to the tissue bed, provides maximum comfort and restores full physiological function to the accessory organs of the eye.
\end{abstract}

Key words: ocular defect; ocular prosthesis; custom made; acrylic prosthesis.

\section{Introduction}

$\mathrm{E}$ yes they say are the 'windows to the soul'. The loss of ocular tissue due to congenital, traumatic, malignancy or radical surgery can affect the psyche of patient adversely. The globe of eye may be removed by enucleation, evisceration, or exenteration. Enucleation is the removal of the globe from orbit, involving the separation of all of the connections between the globe and patient. Evisceration involves the removal of the contents of the eye, while maintaining an intact scleral shell attached to the extraocular muscles. Exenteration involves removal of globe along with all the soft tissues of the orbit. ${ }^{1}$

Before World War II, ocular prostheses were made of glass and supplied in standard sizes and

Conflict of Interest: No

\section{*Corresponding Author}

Dibya Devkota,

Prosthodontist,

Cosmetic Dental Clinic,

E-mail:dibyadevkota72@gmail.com colors that could not be altered. Early in the War, a scarcity of imported glass led to a search for another suitable material. In 1944, investigators at the Naval Graduate Dental School developed a custom-fitted methyl methacrylate resin ocular prosthesis. Currently there are three types of acrylic resin prosthesis used: Stock eyes, stock eyes modified by various methods, and custom-fitted eyes made from an impression of the socket. With stock eyes, the mucosa is easily displaced; some success can be obtained however, the patient will experience some type of discomfort, and the mucosal surface will not fit snugly enough to utilize tissue bed or the implant to their full potential to produce movement. Also, potentially irritating mucus and debris can collect in the voids. Since every socket differs in size and shape, it is obvious that an individually designed prosthesis, made from an impression of the socket, is needed to utilize the full movement potential of tissue bed or implant and also to provide maximum comfort and restore full physiologic function to the accessory organs of the eye. 
Method

\section{History And Clinical Examination}

A 69 year-old female arrived with the chief complaint of a defect in the left eye area. Case history revealed that the patient had traumatic injury 30 years back. A careful examination of the defect showed atrophic remnants of lens and sclera. (Figure 1) The patient was reluctant to undergo any surgery for surgical removal of the remnants of eye (stump). There was no sign of infection or inflammation in the ocular defect, and a custom made ocular prosthesis was planned.

\section{Primary Impression Making:}

The patient was seated in the dental chair, the headrest was adjusted for comfort, and the back rest was reclined slightly. The patient was asked to fix her gaze straight at distant wall, so that the pupil was well centered. Alginate impression material (Zelgan, Dentsply Int.) with excess water was mixed until it was very free flowing, sacrificing strength to avoid tissue distortion. The impression material was loaded in a disposable plastic syringe. The needle and nozzle part of syringe was removed. The lids were gently drawn apart, and the impression material was introduced at the inner side of the palpebral opening, allowing it to flow across the base of the socket and drive out air and lacrimal fluids. Excess material from the syringe was allowed to be ejected over and around the lids. (Figure 2)

\section{Fabrication of Custom-made Ocular Tray and Final Impression}

After the material was set, the impression was gently removed. The impression was checked and custom made ocular acrylic tray was fabricated from it by sprinkle-on method over the impression. After the custom acrylic ocular tray was set, finishing and polishing was done to obtain smooth and rounded finish. Perforations were made to help to retain final impression material. (Figure 3)

The tray was connected to a $5 \mathrm{ml}$ disposable syringe to provide the channel for flow of impression material and final impression was made by injecting light body consistency polyvinyl siloxane elastomer (Reprosil, Dentsply Int) into the eye socket. Patient was instructed to move the eyeball to different directions to ensure the flow of impression material to all fornices and finally gaze at distant wall at eye level. After the material was set, final impression was retrieved from the socket and evaluated for any defects. (Figure 4)

\section{Fabrication of Wax Form and Processing}

The impression was invested in alginate in a disposable glass and the alginate mold was partially split after setting, for retrieving the impression of socket. Molten baseplate wax (Modelling wax, DPI) was poured into the mold to fabricate a scleral wax form. The wax form was then polished. In the next appointment, wax form was checked for proper fit and it was seen that the contour of the corneal prominence matches the adjacent eye giving a similar drape of the eyelid. Adjustments were to obtain the satisfactory contours of eyelids. The adjacent working eye was taken as a reference to mark the iris position on the wax pattern. (Figure $5 \mathrm{a}, 5 \mathrm{~b})$ Measurements were made for correct placement of corneal button medio-laterally and supero-inferiorly. The wax pattern was then polished and trial was done to evaluate its position and gaze. Shade selection of sclera of the natural eye was done. After dewaxing, it was processed in the flask using heat polymerizing acrylic resin (Semident Acrylic Resin, Samit). Thus, the obtained scleral blank was tried in and the supraorbital folds, eyelid margins and iris plane were compared with the contralateral eye. 


\section{Iris Painting and Characterization}

The scleral blank was then painted with acrylic colors (W\&N Artists' Acrylic) so as to match with the color of natural eye. Characterization was enhanced by sprinkling pink acrylic fibers in the sclera to simulate vasculature. (Fig 6) The mold space was scraped to make space for clear acrylic heat cure resin and packing was done. After acrylization, the final obtained prosthesis was finished and polished in order to give a high shine.

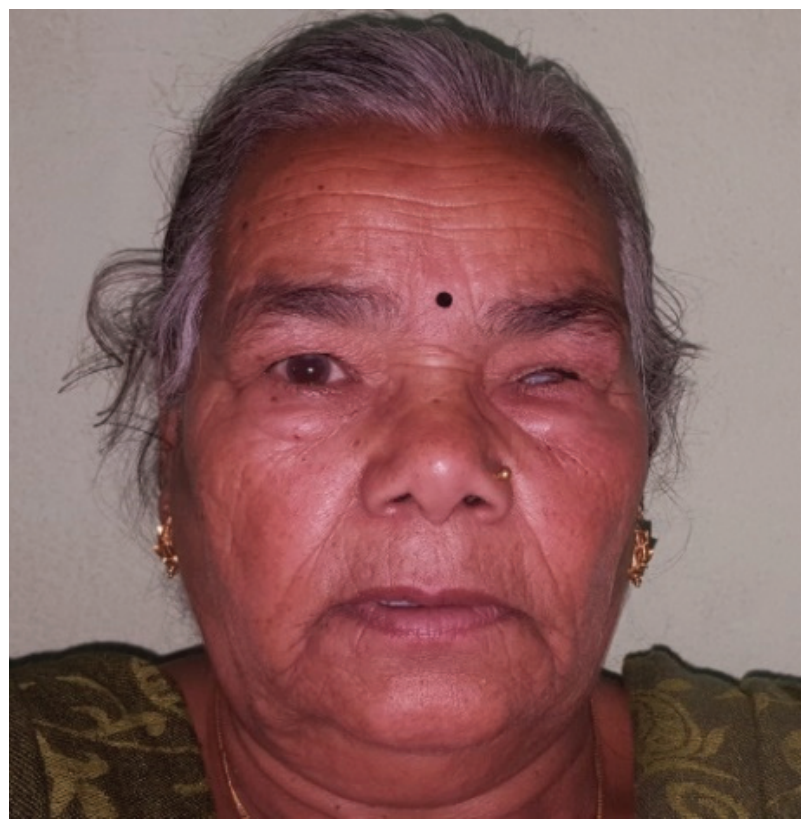

Figure 1: Patient Presentation

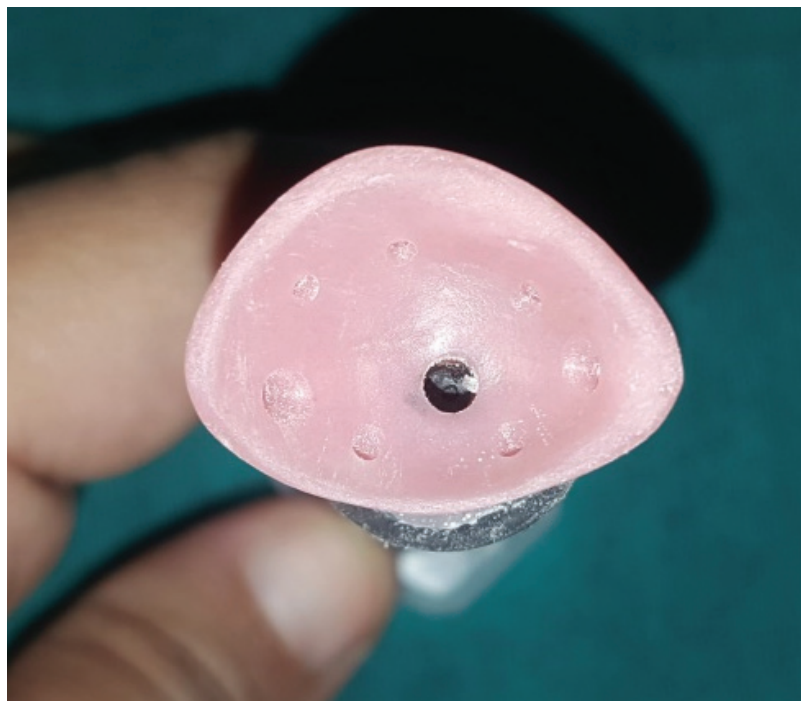

Figure 3: Customized Ocular Impression Tray

\section{Insertion, Post-Insertion Instructions and Follow-Up}

Insertion and patient's approval of the prosthesis was taken and she was trained for insertion and removal. (Fig 7) It was advised to use spectacles for camouflage effect. Post-insertion instructions for hygiene and maintenance were given and follow-up was planned for the coming day and every six months.

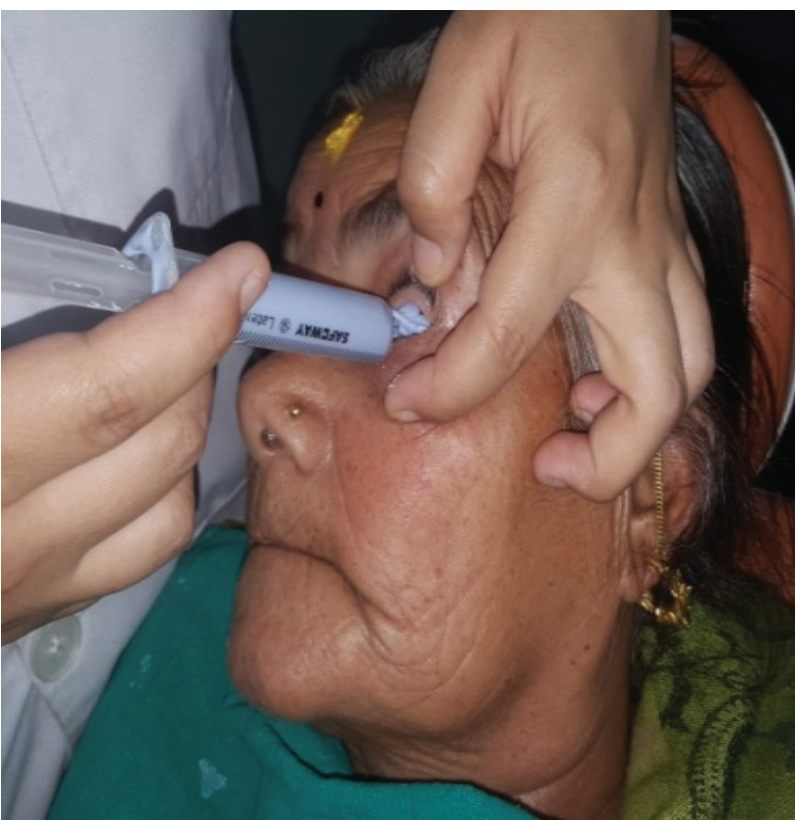

Figure 2: Alginate Impression

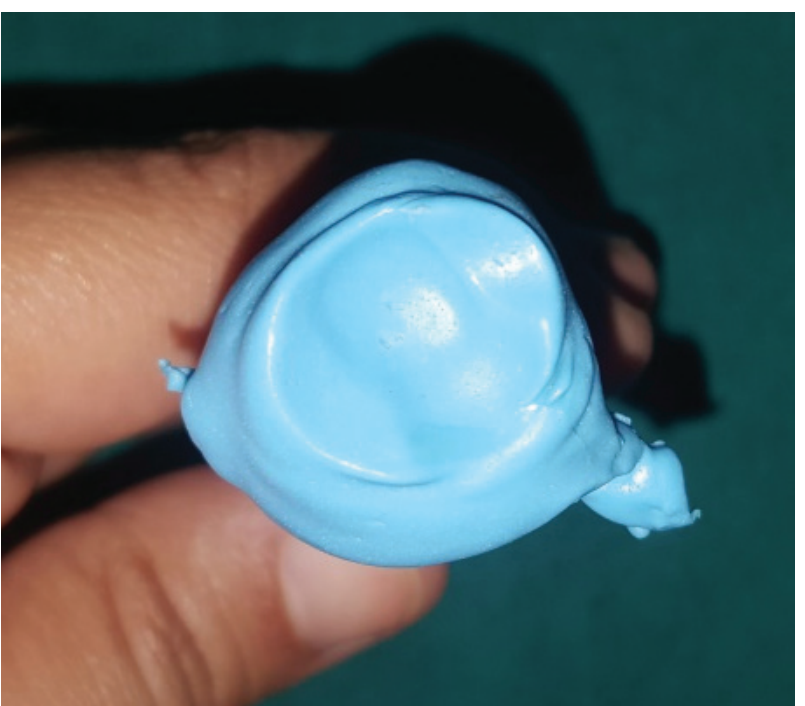

Figure 4: Final Impression 

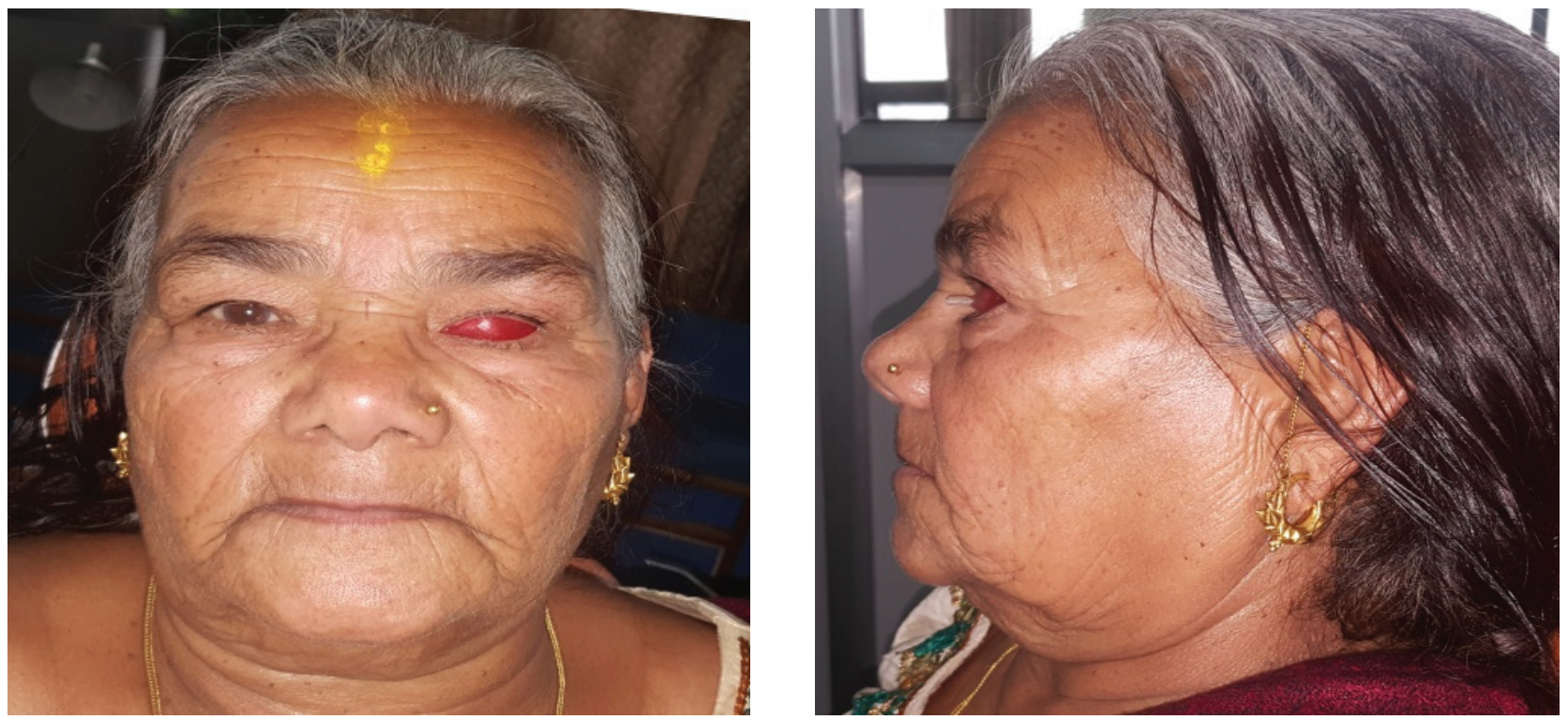

Figure 5a, 5b: Wax Form Trial, Frontal and Sagittal view

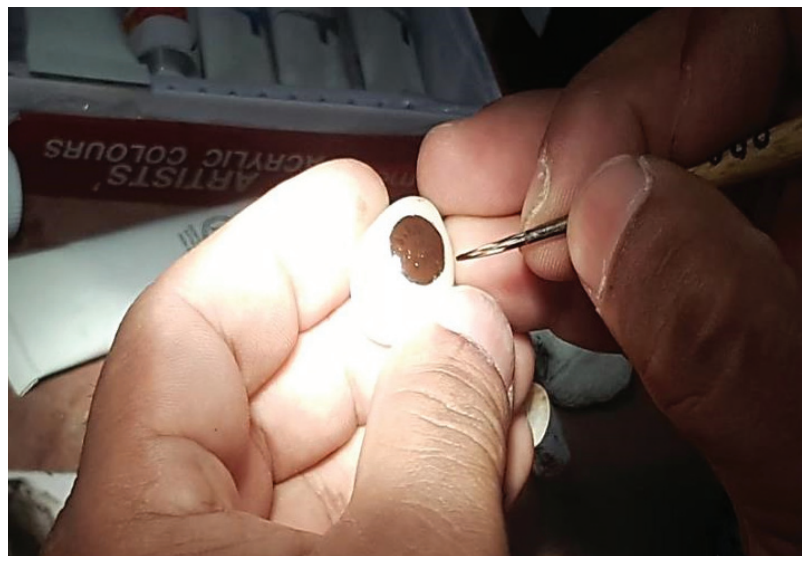

Figure 6: Iris Painting on the sclera blank

\section{Discussion}

Custom-made acrylic resin ocular prosthesis replicates the orientation, natural color, contour, and size of the pupil and iris, providing natural appearance to the patient's face. The close adaptation of custom-made prosthesis tends to distribute pressure more equally than does a stock eye prosthesis. This helps to reduce the incidence of abrasion or ulceration. It also enhances tissue health by decreasing potential stagnation spaces at the prosthetic tissue interface. ${ }^{3}$

A good impression technique can predictably capture the internal tissue surface and fornices of the socket. A number of techniques have

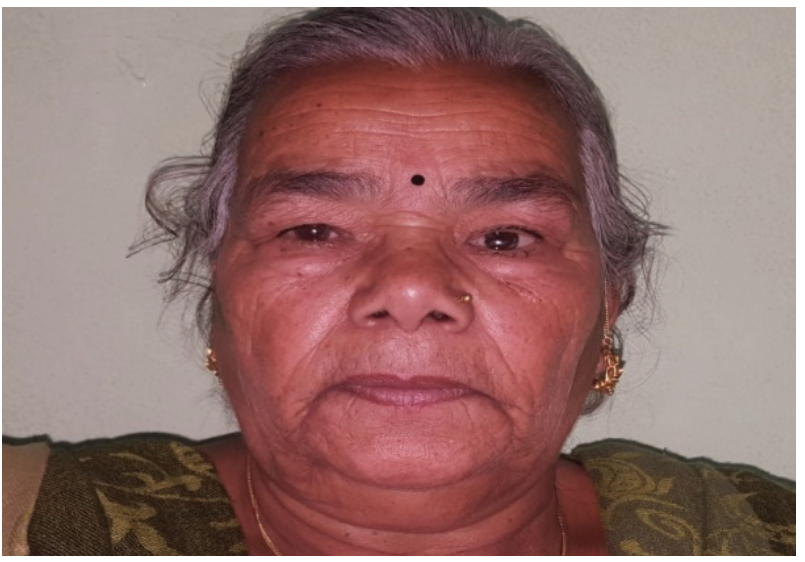

Figure 7: Final Ocular Prosthesis

been advocated for achieving proper impression of anophthalmic socket such as the direct impression/ external impression, use of a stock ocular tray, modification of a stock ocular tray, use of a custom ocular tray, an impression using a stock ocular prosthesis. Basic similarities exist among most techniques. The technique used in above case is a modification in existing methods. It useful in cases where there is limited resources and material. It also skips the process of stone mold formation for the fabrication of custom ocular or wax form. Contrary to conventional method of formation of wax form in stone mold as described by Bartlett and Moore $^{2}$, Brown ${ }^{4}$ and Cain ${ }^{5}$, this technique of investing the impression in alginate impression 
is fast and less technique sensitive. The method of investing impression in alginate for obtaining mold has been described by Methews. ${ }^{6}$

Wax should be roughly egg shaped and congruent with the natural eye. The height of convexity should be centered over the pupil, usually slightly medial to the midline between inner and outer canthi. The palpebral opening should be the same as that of the natural eye. The form of the corneal prominence should match the eye giving a similar drape of the eyelid. The distance from the pupil of the normal eye to the midline is used in establishing the horizontal position of the prosthetic pupil center and is marked on the wax form. The vertical position of the pupil center is determined and marked by the canthus relationship. Holding a ruler close to the normal steadied eye, the diameter of the iris is measured. The wax form with its marked pupillary center is removed and the iris size is circumscribed with a compass from the established point. A preformed disk that is 1 $\mathrm{mm}$ smaller than the diameter of the patient's iris is selected. This will compensate for the magnification caused by the overlay of clear acrylic resin in the completed prosthesis. in this case, iris positioning was done in wax form rather than in acrylic scleral blank as described by Brown. ${ }^{4}$

For painting of the iris appropriate acrylic colors are mixed with mono-poly to form colored syrup. The colors are applied with a small paintbrush in a circular pattern radiating outward from the center. The darkest base shades are used first, progressing to the lighter individual colors. The collarette and limbus are painted last. Striations are developed in the pigments by using light strokes in overlaying the different colors. It is possible with this technique to create a three-dimensional effect. After drying, the mold is scraped for space for clear acrylic and subsequent packing, curing and finishing is done. Thus, obtained custom eye prosthesis will meet the psychological demand of patient, improving confidence and self-esteem.

\section{Conclusion}

Rehabilitation of ocular defect is a challenging task and requires individualized tailoring of the technique for each patient. The method of choice is largely governed by the type of defect, operator skills and availability of material and equipment. The careful sequence of preparation of customized ocular prosthesis ensures a better drape of lid tissues, and provides superior natural appearance, improved hygiene and patient satisfaction.

\section{References}

1. Beumer J, Marunick MT, Esposito SJ. Maxillofacial rehabilitation : prosthodontic and surgical management of cancer-related, acquired, and congenital defects of the head and neck. Hanover Park, IL: Quintessence Pub.; 2011.

2. Bartlett SO, Moore DJ. Ocular prosthesis: a physiologic system. J Prosthet Dent. 1973;29(4):450-9.

3. Jayaprakash M, Misra S. The ocular impression: a review. J Dent. 2014;1(2):61-3.

4. Brown KE. Fabrication of an ocular prosthesis. J Prosthet Dent. 1970;24(2):225-35.

5. Cain JR. Custom ocular prosthetics. J Prosthet Dent. 1982;48(6):690-4.

6. Mathews MF, Smith RM, Sutton AJ, Hudson R. The ocular impression: A review of the literature and presentation of an alternate technique. $\mathrm{J}$ Prosthodont. 2000;9(4):210-6. 